Recepción: 13 / 02 / 2018

Aceptación: 09 / 04 / 2018

Publicación: 01 / 08 / 2018

(c) (†) (이 Ciencias económicas y empresariales

Artículo de investigación

\title{
Marketing digital una nueva estrategia para los emprendedores
}

\author{
Digital marketing a new strategy for entrepreneurs
}

Marketing digital uma nova estratégia para empreendedores

\author{
Mónica P. Salazar-Tapia ${ }^{I}$ \\ monica.salazar8191@utc.edu.ec \\ Nelly G. Salguero-Barba II \\ nelly.salguero@utc.edu.ec \\ Christian P. García-Salguero III \\ crispagas@hotmail.com
}

Correspondencia: monica.salazar8191@utc.edu.ec

I Especialista en Creación de Empresas, Magister en Dirección de Empresas, Ingeniera Informática, Docente de la Universidad Técnica de Cotopaxi, Latacunga, Ecuador.

II Magister en Planeamiento y Administración Educativos, Diploma Superior en Didáctica de la Educación Superior, Licenciada en Administración Ejecutiva, Docente de la Universidad Técnica de Cotopaxi, Latacunga, Ecuador.

III Docente Universidad de Toronto, Toronto, Canadá. 


\title{
Resumen
}

El marketing digital es una nueva estrategia que se usa en las empresas para tener presencia online, incremento en las ventas y posicionamiento de marca por medio de la evolución de herramientas tecnológicas, cuyo objetivo es obtener rapidez en las respuestas en tiempo real y construir una comunidad virtual, donde el usuario se convierte en actor principal; interactúa en la red a través de medios digitales como Facebook, twitter, foros, blogs, videos, pagina web, chats de tal forma que el internauta sea escuchado, es necesario realizar un estudio de todas las herramientas digitales para seleccionar la que mejor se adapte a la empresa. En la presente investigación se utilizó la metodología de trabajo, a través del Análisis inicial de la identidad digital, la misma que será el punto de partida para saber a dónde queremos llegar y cómo conseguirlo, conociendo el posicionamiento del sitio Web y redes sociales. En conclusión, podemos decir que el empresario puede monitorear el mercado, mediante la aplicación de estrategias digitales, las mismas que pueden utilizar los emprendedores sin ningún costo; debido a que el internet ofrece las herramientas tecnológicas de manera gratuita, con la finalidad de obtener ROI.

Palabras Claves: redes sociales; marketing en línea; internet; sitio web; empresas.

\begin{abstract}
Digital marketing is a new strategy that is used in companies to have an online presence, increase in sales and brand positioning through the evolution of technological tools, whose goal is to obtain quick responses in real time and build a community virtual, where the user becomes the main actor; interacts in the network through digital media such as Facebook, twitter, forums, blogs, videos, web page, chats in such a way that the Internet user is listened to, it is necessary to carry out a study of all the digital tools to select the one that best suits to the enterprise. In this research work methodology was used, through the initial analysis of digital identity, which will be the starting point to know where we want to get and how to get it, knowing the positioning of the website and social networks. In conclusion we can say that the employer can monitor the market, through the application of digital strategies, which can be used by entrepreneurs at no cost; because the internet offers the technological tools for free, in order to obtain ROI.
\end{abstract}

Keywords: social networks; online marketing; internet; website; business. 


\section{Resumo}

Marketing digital é uma nova estratégia que é usada no negócio para ter presença online, aumentar as vendas e posicionamento da marca através do desenvolvimento de ferramentas tecnológicas, com o objetivo de fornecer respostas rápidas em tempo real e construir uma comunidade virtual, onde o usuário se torna o ator principal; interage rede através de meios digitais como Facebook, Twitter, fóruns, blogs, vídeos, páginas web, chats para que o usuário da Internet é ouvido, é necessário realizar um estudo de todas as ferramentas digitais para escolher aquele que melhor se adequa para a empresa. A metodologia de trabalho utilizada nesta investigação através da análise inicial da identidade digital, que será o ponto de partida para saber para onde queremos ir e como obtê-lo, sabendo a posição do site e redes sociais. Em conclusão, podemos dizer que o empreendedor pode monitorar o mercado, através da aplicação de estratégias digitais, as mesmas que os empreendedores podem utilizar sem custo algum; porque a internet oferece as ferramentas tecnológicas de graça, a fim de obter o ROI.

Palavras chave: redes sociais; marketing on-line; internet; site web; empresas.

\section{Introducción}

A partir de la evolución del Internet los emprendedores están expandiendo sus negocios, la tecnología está cambiando frecuentemente, para lo cual es necesario adaptarse a los cambios, generar nuevas ideas de negocios, desarrollar estrategias competitivas involucradas en la era digital. El internet ha ido evolucionando continuamente, la web aparece en 1996, había 45 millones de usuarios y 250.000 sitios estas webs eran solo de lectura, posteriormente aparece la web 2.0 en el 2006 existen más de 1 billón de usuarios y 80.000.000 sitios, esta web era de lectura y escritura. (Domene Maciá Fernando y Grela Javier Gosende, 2013, p.33). Con este tipo de información se observa que en la actualidad se puede leer y escribir; es decir que el usuario sea activo ya que participa en los canales de comunicación y en los medios digitales, puede comentar, compartir información, fotos y videos convirtiendo al usuario final como un actor.

El mundo del internet es amplio, razón por la cual es importante estar preparado para tener éxito en el negocio, innovar frecuentemente, ser muy creativo, adaptarse rápidamente a los cambios, conocer la competencia y el mercado objetivo.

Las empresas tradicionales deberían migrar sus negocios para que tengan presencia en el internet y los emprendedores deberían tomar en cuenta este tipo de estrategias al crear el negocio. El 
marketing digital es la estrategia que se está tomando más énfasis en la actualidad, cambiando el marketing tradicional a la digital.

\section{Importancia del problema}

En la actualidad existen muchas personas que necesitan realizar una compra de un producto o servicio desde cualquier lugar y a cualquier hora, mediante el uso del teléfono celular y el internet; por lo cual las empresas al no tener presencia online se están perdiendo muchas ventas. Es importante que los emprendedores consideren al marketing digital como una nueva estrategia para sus emprendimientos.

Otro tipo de problema que se tiene es la existencia de miles de sitios web en el internet; una de los objetivos es lograr que aparezca en las primeras posiciones de búsqueda de google.

Los usuarios finales han adquirido nuevos hábitos de consumo debido a que frecuentemente utilizan el celular. Existen muchas ventajas competitivas el tener presencia en internet, ya que se puede realizar pedidos las 24 horas del día sin estar presente. Marketing Online no solo implica tener un sitio web, sino que se debe realizar estrategias para que sea encontrado el emprendimiento.

Actualizar frecuentemente los productos y servicios en internet similar a una tienda física. Transmitir confianza al usuario final para que tome la decisión de compra.

Existen muchos navegadores en el internet como son Google, Yahoo, Bing; pero el buscador que es más utilizado por los usuarios es google; razón por la cual las estrategias de marketing deben ser aplicado para este navegador.

En el Internet existen miles de emprendimientos con los cuales se va a competir, lo importante es realizar el estudio y aplicar las estrategias adecuadas. Actualmente hay muchos emprendimientos digitales que no tienen bien definido los objetivos, hacia donde se dirigen.

\section{Metodología}

Se realiza un análisis inicial mediante estudio de la competencia, mercado objetivo, gustos preferencias, herramientas tecnológicas. Mediante el análisis poder establecer las estrategias adecuadas; ya que existen variedad de herramientas en el internet gratuitas y pagadas, tomar decisiones de acuerdo a varios parámetros como, por ejemplo: tipo de empresa, tamaño de la empresa, mercado objetivo. Conocer las estadísticas desde un inicio y realizar comparaciones en 
el futuro, con esto nos ayuda a saber si la estrategia tiene éxito o de lo contrario cambiar de estrategia.

\section{Resultados}

Con los resultados obtenidos en el análisis (estadísticas) se espera aplicar las herramientas adecuadas, tomando en cuenta los costos, mercado objetivo, competencia, tamaño del emprendimiento, ubicación para obtener un ROI Return On Investment (Retorno de la inversión) adecuado al aplicar las estrategias de internet. La ventaja es que se puede analizar en tiempo real si la estrategia tiene éxito o no.

\section{Discusión}

Lo importante al aplicar estrategias de marketing digital es lograr calidad de tráfico, no cantidad, porque puede haber muchas visitas, pero sin interés, en cambio sí ingresan pocos son las personas que realmente tienen interés de compra.

Mediante el análisis se podrá identificar como atraer tráfico de calidad hacia un sitio web o hacia una página de redes sociales para posicionar en google, se podrá medir las estadísticas y mejorar para obtener mejores conversiones en las ventas, identificar las mejores herramientas para poder fidelizar a los clientes e identificar cuales redes sociales utilizan el mercado objetivo al que se requiere aplicar las estrategias; después obtener estadísticas para saber que está pasando y luego invertir en la herramienta más adecuada. Las estrategias de marketing digital requieren de cierto tiempo para lograr efectividad.

\section{Conclusión}

En la actualidad, se debe adaptar los emprendimientos de acuerdo a los avances tecnológicos para obtener más rentabilidad. Identificar los perfiles del mercado objetivo, establecer las mejores estrategias SEO Search Engine Optimization (Optimización en buscadores), SEM Search Engine Marketing (Marketing en buscadores), correo electrónico, redes sociales con la finalidad de atraer visitas, luego para convertirlos en clientes y posteriormente fidelizarlos. 


\section{Referencias Bibliográficas}

Domene Maciá Fernando y Grela Javier Gosende, 2013, Marketing Online Estrategia para ganar clientes en Internet, Madrid: Sexta edición.

López Gómez Miguel, 2008, Marketing Online Posicionamiento en Buscadores. www.consultoresvalencia.com.

\section{Anexos}

Gráfico 1. Muestra cómo se mide los resultados de la estrategia aplicada en tiempo real

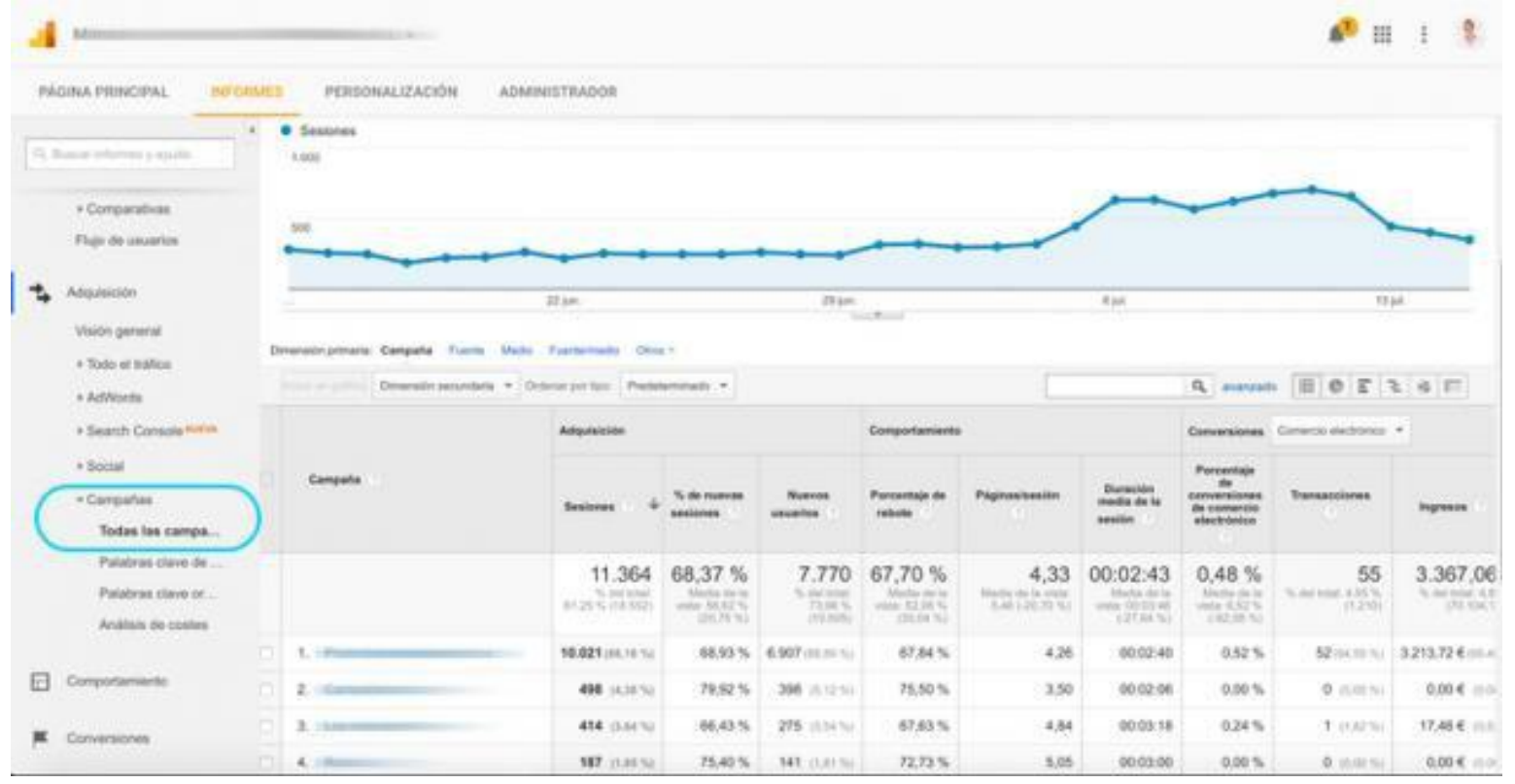

Fuente: Autor 
Gráfico 2. Muestra un diagnóstico de un sitio web con SEOquake

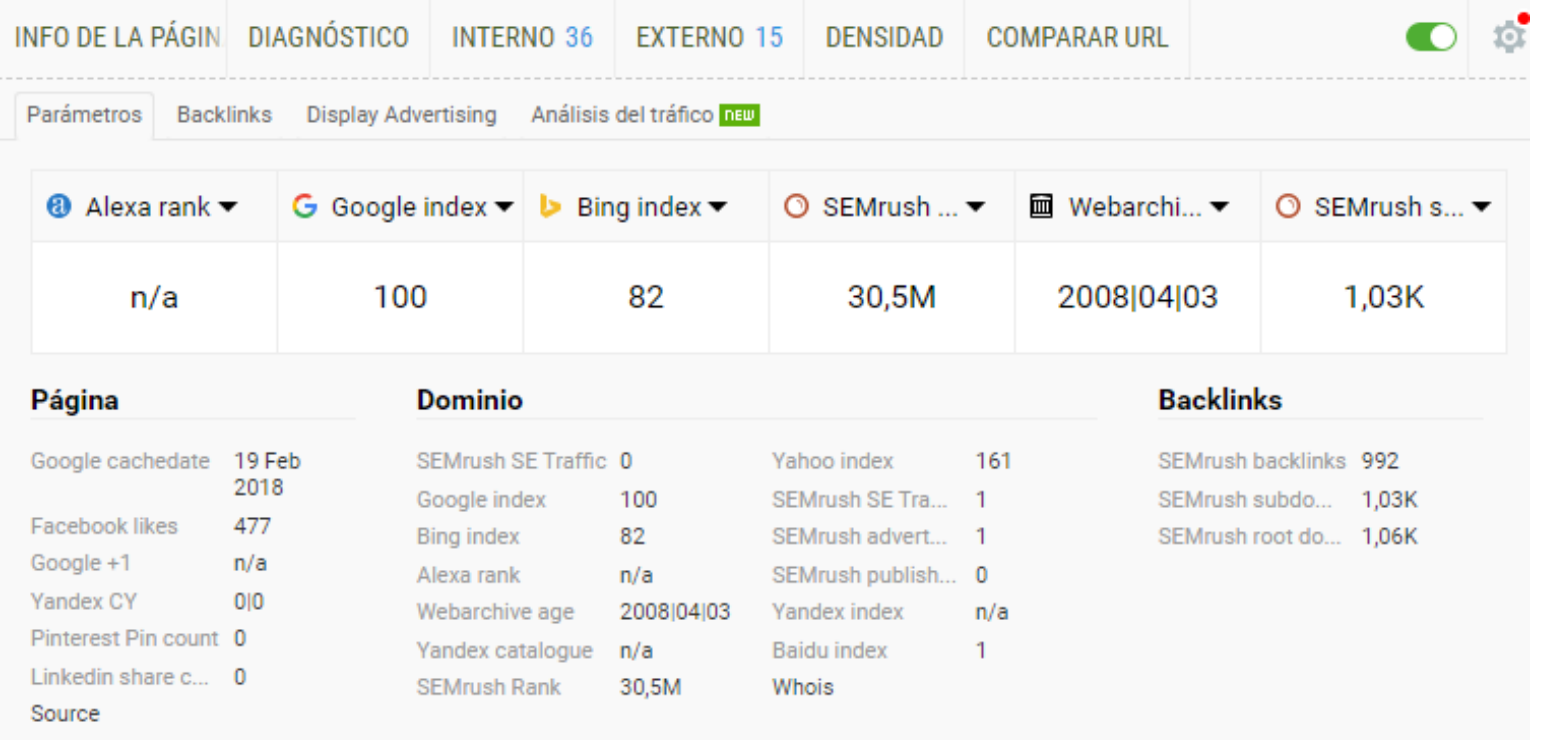

Fuente: Autor 